\title{
Role of $\mathrm{Gd}_{2} \mathrm{O}_{3}$-doped carbon-11-choline-lenvatinib nanoparticles contrast agent PET/CT in the diagnosis of patients with lung cancer
}

\author{
TONG ZHOU ${ }^{1-3}$, DONGFANG HANG ${ }^{1-3}$, YING LI $^{1-3}$, JIN ZHANG $^{1-3}$, \\ HUAYANG WU ${ }^{1-3}$, HONGYAN WANG ${ }^{1-3}$, ENBING TIAN ${ }^{1-3}$ and JUN YAN ${ }^{1-3}$ \\ ${ }^{1}$ Department of Nuclear Medicine, Dongzhimen Hospital of Beijing University of Traditional Chinese Medicine; \\ ${ }^{2}$ Department of Oncology, Beijing Chao-Yang Hospital Affiliated to Capital Medical University; \\ ${ }^{3}$ Department of Respiratory Medicine, Dongzhimen Hospital of Beijing University \\ of Traditional Chinese Medicine, Beijing 100700, P.R. China
}

Received September 26, 2018; Accepted May 31, 2019

DOI: $10.3892 / \mathrm{ol} .2019 .11243$

\begin{abstract}
Positron emission tomography-computed tomography $(\mathrm{PET} / \mathrm{CT})$ is an efficient method for the diagnosis of various types of human cancer. Studies have demonstrated that $\mathrm{Gd}_{2} \mathrm{O}_{3}$-doped carbon-11-choline (GdCho) can be used as a contrast nanoparticle for PET/CT in the diagnosis of patients with lung cancer. The aim of the present study was to evaluate the effect of GdCho-lenvatinib nanoparticles contrast-PET/CT (GdCho-Len-PET) in the diagnosis and treatment planning of a cohort of patients suspected of having lung cancer. The results of the present study demonstrated that GdCho-Len could be used as an efficient PET/CT contrast agent for the diagnosis of patients with lung cancer. GdCho-Len nanoparticles contrast agent exhibited a significantly improved longitudinal relaxivity compared with GdCho. The outcomes of the present study were that GdCho-Len-PET diagnosed 152 patients with lung cancer, whereas GdCho-PET diagnosed 130 patients with lung cancer among the 172 patients. GdCho-Len-PET presented with higher accuracy and sensitivity compared with GdCho-PET in diagnosing patients with lung cancer. All patients were further confirmed via histological analysis. GdCho-Len-PET contributed to the anticancer treatments in 56 out of $62(90.3 \%)$ patients with lung cancer who were candidates for radiation therapy, 52 out of 57 (91.2\%) patients with lung cancer undergoing adjuvant radiotherapy, and 13 out
\end{abstract}

Correspondence to: Professor Jun Yan, Department of Nuclear Medicine, Dongzhimen Hospital of Beijing University of Traditional Chinese Medicine, 5 Haiyuncang Road, Dongcheng, Beijing 100700, P.R. China

E-mail: yanjunbeijing@yeah.net

Key words: lung cancer, positron emission tomography-computed tomography, $\quad \mathrm{Gd}_{2} \mathrm{O}_{3}$-doped carbon-11-choline-lenvatinib nanoparticles contrast-positron emission tomography-computed tomography of $17(76.5 \%)$ patients with lung cancer undergoing comprehensive therapy. Patients diagnosed using GdCho-Len-PET improved the survival of patients with lung cancer during a 420-day follow up. In conclusion, GdCho-Len-PET increased the diagnostic efficacy and had a significant effect on survival for patients with lung cancer, and may therefore serve as a reliable method for human cancer diagnosis.

\section{Introduction}

Lung cancer is a major public health problem and is the leading cause of cancer-associated mortality worldwide (1-3). Cancer pathology often divides lung cancer into non-small cell lung cancer (NSCLC) and small cell lung cancer, which account for $\sim 85$ and $\sim 15 \%$ of lung cancer cases, respectively (4). Statistics have estimated that there were $\sim 1.8$ million newly diagnosed lung cancer cases and $\sim 1.6$ million lung cancer-associated mortalities in 2012 worldwide (5). Lung cancer is the most frequently occurring human cancer and is the leading cause of cancer-associated mortality among males, followed by prostate and colorectal cancer for incidence, and liver and stomach cancer for mortality (6). Currently, although clinical therapeutic methods, including radiotherapy, chemotherapy, Chinese medicinal herb treatment, immunotherapy, gene therapy and targeted therapy, have been investigated and applied for the treatment of patients with lung cancer (7-10), the overall 5-year survival rate remains poor at $<15 \%(11-13)$.

At present, lung tumor metastasis is the most difficult treatment barrier in cancer therapy (14-16). Therefore, obtaining an early diagnosis for human tumors is crucial for the effective treatment of human lung cancer (17). Clinically, ultrasound, positron emission tomography-computed tomography (PET/CT) and magnetic resonance imaging have been applied for diagnosing human cancer (18). Notably, PET/CT has become an efficient protocol for tumor diagnosis in human lung cancer cases (19-21). PET/CT also serves a vital role in the differentiation of adrenal metastasis from a benign adrenal mass in patients with lung cancer, with excellent diagnostic 
performance (22). However, the diagnostic efficacy in patients with early-stage lung cancer requires improvement.

It has been reported that developing multimodal contrast agent would enhance the diagnostic accuracy of PET/CT, as well as increase the diagnostic accuracy sensitivity in patients with lung cancer (23). A previous study reported that contrast-enhanced ultrasound with a novel nanoparticle contrast agent increases the diagnostic efficacy in patients with NSCLC (24). In addition, another study reported a composite nano-system composed of gadolinium-doped mesoporous silica nanoparticles and gold nanoparticles, which can be used as an efficient contrast agent for in vivo cancer imaging (25). In addition, previous studies have demonstrated that $\mathrm{Gd}_{2} \mathrm{O}_{3}$-doped nanoparticles are promising candidates of highly efficient contrast agents in diagnosing human cancer (26-28).

Lenvatinib (Len) is a small-molecule tyrosine kinase inhibitor that inhibits vascular endothelial growth factor receptors, platelet-derived growth factor receptor $\alpha$, fibroblast growth factor receptors, stem cell factor receptor and rearranged during transfection (29). In the present study, $\mathrm{Gd}_{2} \mathrm{O}_{3}$-doped carbon-11-choline-Len (GdCho-Len) nanoparticles contrast combined with PET/CT (GdCho-Len-PET) was used to diagnose patients with lung cancer. The present study characterized GdCho-Len-PET to visualize the distribution of human lung tumor using PET/CT by performing in vivo trails. The survival rate of patients with lung cancer diagnosed by GdCho-Len-PET was identified during a 420-day follow up.

\section{Materials and methods}

Subjects. A total of 172 patients with suspected lung cancer were recruited from the Dongzhimen Hospital of Beijing University of Traditional Chinese Medicine (Beijing, China) between May 2016 and September 2017. Lung cancer diagnosis was confirmed by biopsy by three respiratory physicians who specialized in the interpretation of clinical and radiological lung cancer data. All patients with suspected lung cancer underwent GdCho-PET and GdCho-Len-PET, which was further confirmed by a tissue biopsy $(n=172)$. The age range of patients was 36-60 years, and comprised an equal number of men and women. The characteristics of the patients are summarized in Table I. The exclusion criteria were as follows: i) Patients with cancer history; ii) patients with pulmonary infarction; iii) patients who had been diagnosed with acute respiratory disease within 6 months; iv) pregnant or lactating females; and v) patients with infection suspected to cause coughs. The inclusion criteria were as follows: i) age $\geq 25$ years; and ii) individuals who were able to provide informed consent for participation. The Ethics Committee of the Dongzhimen Hospital of Beijing University of Traditional Chinese Medicine (Beijing, China) approved the present study. All participants provided written informed consent for inclusion.

Contrast agent. The GdCho and GdCho-Len contrast agents were synthesized as described previously (30). Briefly, cetyltrimethylammonium bromide $\left(\mathrm{C}_{16} \mathrm{TAB} ; 0.2 \mathrm{~g}\right)$ was dissolved in distilled water $(50 \mathrm{ml})$. Subsequently, $\mathrm{NH}_{3} \cdot \mathrm{H}_{2} \mathrm{O}(2 \mathrm{ml}$; $25 \%)$ and tetraethoxysilane $(4.49 \mathrm{mmol})$ were added and stirred at room temperature for $10 \mathrm{~min}$. $\mathrm{Gd}_{2} \mathrm{O}_{3}(0.5 \mathrm{mmol})$
Table I. Characteristics of patients with suspected lung cancer.

\begin{tabular}{lcc}
\hline Characteristics & $\mathrm{n}(\%)$ & $\begin{array}{c}\text { Mean } \pm \text { standard } \\
\text { deviation }\end{array}$ \\
\hline Sex & & \\
Male & $86.0(50.0)$ & \\
Female & $86.0(50.0)$ & \\
Age, years & & \\
Mean & 47.6 & $26.2 \pm 5.6$ \\
Range & $36.0-60.0$ & \\
BMI & \\
Heart rate, beats/min & \\
Smoking status & & \\
Current/former & $160.0(93.0)$ & \\
Never & $12.0(7.0)$ & \\
\hline
\end{tabular}

BMI, body mass index.

was then added to the solution and stirred at room temperature for $1 \mathrm{~h}$, and carbon-11 $(0.1 \mathrm{mmol})$, choline $(0.1 \mathrm{mmol})$ or carbon-11-choline $(0.1 \mathrm{mmol})$, and lenvatinib $(0.2 \mathrm{mmol})$ were added to the solution and stirred at room temperature for $1 \mathrm{~h}$. All these compounds were provided by Sigma-Aldrich; Merck KGaA. Samples were calcined at $37^{\circ} \mathrm{C}$ for $72 \mathrm{~h}$, and the GdCho and GdCho-Len nanoparticles were harvested. The synthesized GdCho-Len nanoparticles were imaged by high-angle annular dark-field scanning electron microscopy (magnification, x100). The size distribution of the GdCho-Len nanoparticles was measured using a DynaPro NanoStar Dynamic Light Scattering Detector (Wyatt Technology Corporation). The nanoparticles contrast agent was visualized by a PET/CT system. The GdCho and GdCho-Len contrast agents were intravenously injected prior to PET/CT.

$P E T / C T$. Static PET/CT with a GEMINI TF Big Bore PET/CT system (Philips Medical Systems, Inc.) was used to evaluate patients with suspected lung cancer. PET/CT was performed at $3 \mathrm{~h}$ following the administration of GdCho-Len (0.4-4.0 mg/kg; $0.4 \mathrm{mg}$ interval). A low dose CT of $30 \mathrm{sec}$ (mAs, 80-175; kV, 120; slice thickness, $5 \mathrm{~mm}$ ) was performed and CT images were set at a 512 matrix. The emission time per bed position ranged between 1 and 2 min based on the body mass index of individuals.

Detection of GdCho-Len in plasma concentration. The serum concentration levels of Len were analyzed using an ELISA kit (cat. no. FAB357P; R\&D Systems, Inc.), according to the manufacturer's protocol. The results were analyzed using an ELISA reader system (1775xMark ${ }^{\mathrm{TM}}$; Bio-Rad Laboratories, Inc.).

Hematoxylin and eosin staining. Biopsies of lung tissues were obtained from individuals following diagnosis by GdCho-Len-PET or GdCho-PET. Sections $4-\mu$ m-thick were prepared, fixed with $10 \%$ paraformaldehyde for $15 \mathrm{~min}$ at room temperature and stained with hematoxylin and eosin 


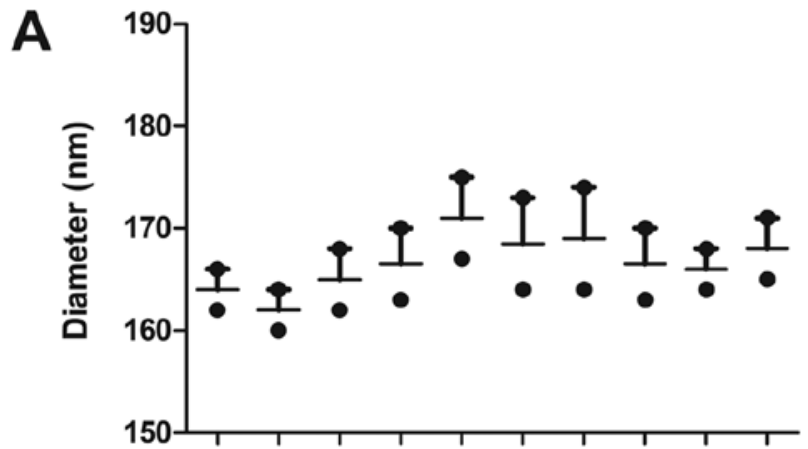

GdCho-Len

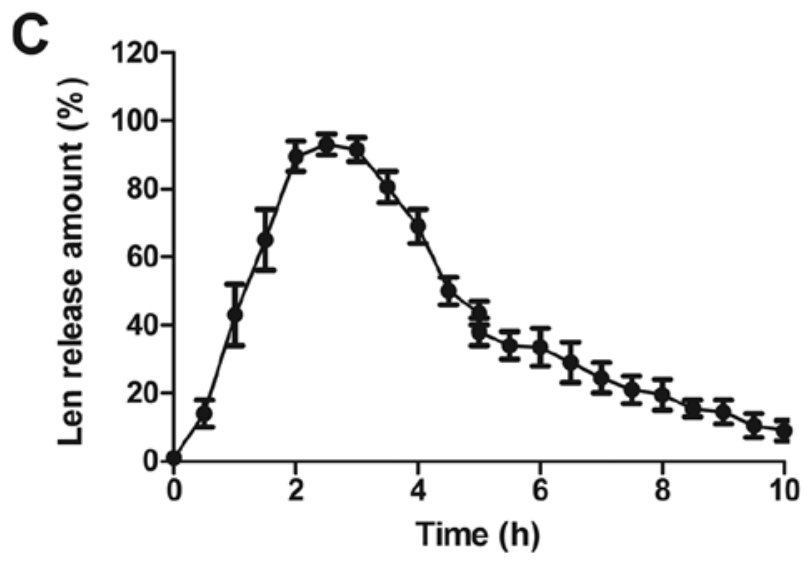

B

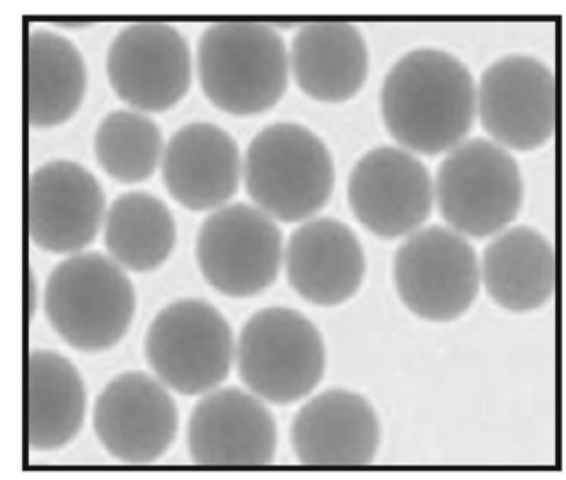

GdCho-Len

D

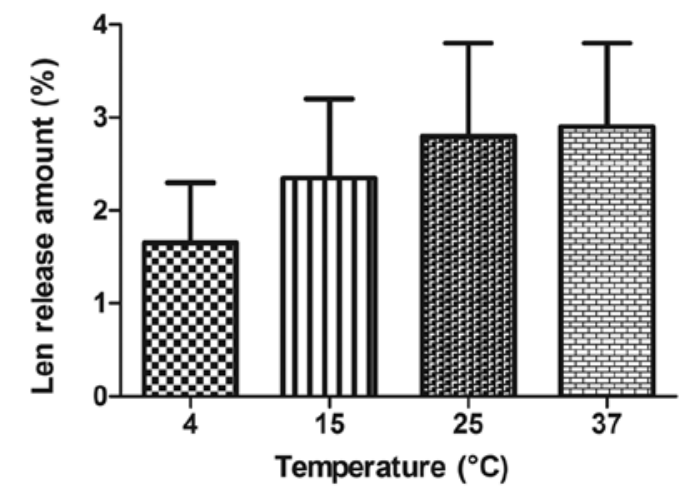

Figure 1. Characterization of GdCho-Len. (A) Diameter of GdCho-Len. (B) The spherical and uniform shape of GdCho-Len. Magnification, x100. (C) Release assay of Len from GdCho-Len. (D) The stability of GdCho-Len nanoparticles at 4, 15, 25 and $37^{\circ} \mathrm{C}$. GdCho-Len, $\mathrm{Gd}_{2} \mathrm{O}_{3}$-doped carbon-11-choline-lenvatinib nanoparticles contrast; Len, lenvatinib.

for $30 \mathrm{~min}$ at room temperature. Sections were washed with PBS three times and then observed under a light microscope (Olympus Corporation; magnification, x100).

Stability assay. GdCho-Len nanoparticles were placed at $4,15,25$ and $37^{\circ} \mathrm{C}$ for 7 days. Stability of GdCho-Len was analyzed by high performance size exclusion chromatography performed using a TSKgel G3000SWxl column (Tosoh Bioscience) and an Agilent HPLC 1200 system (Agilent Technologies Gmbh).

Statistical analysis. Statistical analyses were analyzed using SPSS 18.0 software (SPSS, Inc., Chicago, IL, USA). Data are presented as the mean \pm standard error of the mean. All experiments were repeated at least three times. A receiver operator characteristic curve was generated to determine the cut-off point that optimized sensitivity and specificity. A paired Student's t-test was used to compare two independent groups of data. Survival curves were constructed using the Kaplan-Meier method and were compared using a log-rank test. $\mathrm{P}<0.05$ was considered to indicate a statistically significant difference.

\section{Results}

Characterization of GdCho-Len. TEM revealed that the diameter of GdCho-Len was $168.2 \pm 6.8 \mathrm{~nm}$ (Fig. 1A). As presented in Fig. 1B, GdCho-Len exhibited a spherical and uniform shape. The in vitro release of Len from the GdCho-Len was also investigated to determine its release profile (Fig. 1C). The stability assay demonstrated that GdCho-Len nanoparticles were stable particles at $4,15,25$ and $37^{\circ} \mathrm{C}$ for multiple laser irradiations (Fig. 1D). These results indicate the successful encapsulation of Len into the GdCho, and GdCho-Len was demonstrated to be a stable nanoparticles contrast agent.

Diagnostic efficacy of GdCho-Len-PET in patients with suspected lung cancer. The diagnostic accuracy and sensitivity of GdCho-Len-PET was investigated in patients with suspected lung cancer. A clinical dose of GdCho-Len at $2.4 \mathrm{mg} / \mathrm{kg}$ was identified to achieve the optimum signal intensity for PET/CT detection (Fig. 2A). GdCho-Len nanoparticles contrast agent exhibited a markedly improved longitudinal relaxivity compared with GdCho (Fig. 2B). The results indicated that GdCho-Len-PET diagnosed 152/172 patients with lung cancer, while GdCho-PET diagnosed 130/172 patients with lung cancer (Table II), and that GdCho-Len-PET has higher accuracy and sensitivity compared with GdCho-PET in diagnosing patients with lung cancer (Fig. 3).

Histopathological diagnoses of patients with lung cancer. Immunohistochemistry was used to confirm the diagnostic outcomes of GdCho-Len-PET. Fig. 4 presents representative cancer and non-cancer tissues. Statistical analysis demonstrated 
Table II. Diagnostic outcomes of GdCho-Len-PET and GdCho-PET.

\begin{tabular}{lccc}
\hline Presence of lung cancer & GdCho-PET, n $(\%)$ & GdCho-Len-PET, n $(\%)$ & P-value \\
\hline Lung cancer & $130(75.6)$ & $152(88.4)$ & 0.035 \\
No lung cancer & $42(24.4)$ & $20(11.6)$ & 0.023
\end{tabular}

GdCho-Len-PET, $\mathrm{Gd}_{2} \mathrm{O}_{3}$-doped carbon-11-choline-lenvatinib nanoparticles contrast combined with positron emission tomography-computed tomography; GdCho-PET, $\mathrm{Gd}_{2} \mathrm{O}_{3}$-doped carbon-11-choline nanoparticles contrast combined with positron emission tomography-computed tomography.
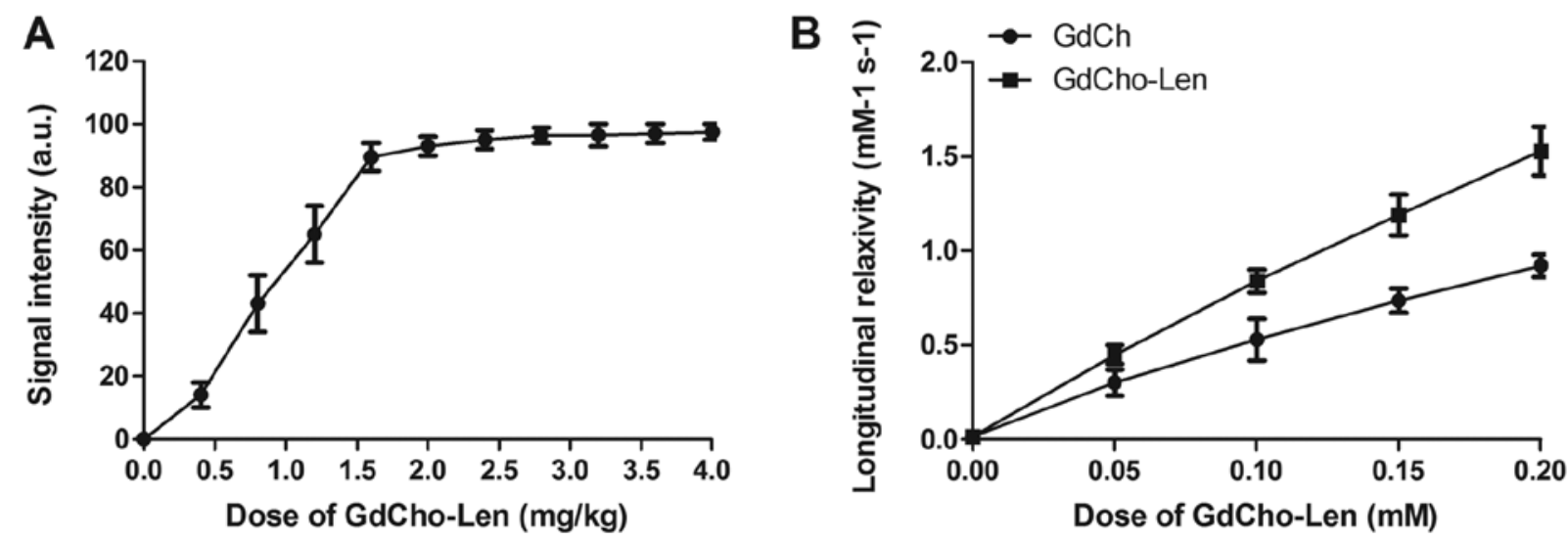

Figure 2. Diagnostic efficacy of GdCho-Len-PET in patients with suspected lung cancer. (A) Signal intensity of GdCho-Len at different concentrations in diagnosing patients with suspected lung cancer. (B) Longitudinal relaxivity of GdCho-PET at different concentrations. $\mathrm{GdCh}_{2} \mathrm{Gd}_{2} \mathrm{O}_{3}$-doped carbon-11; GdCho-Len, $\mathrm{Gd}_{2} \mathrm{O}_{3}$-doped carbon-11-choline-lenvatinib nanoparticles contrast; GdCho-Len-PET, $\mathrm{Gd}_{2} \mathrm{O}_{3}$-doped carbon-11-choline-lenvatinib nanoparticles contrast combined with positron emission tomography-computed tomography.

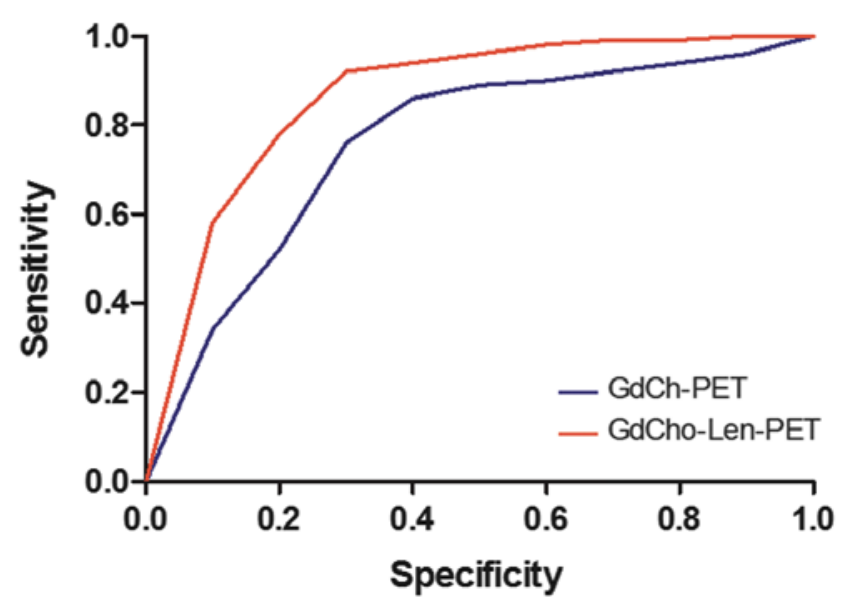

Figure 3. Accuracy and sensitivity of GdCho-Len-PET and GdCho-PET in diagnosing patients with suspected lung cancer. Receiver operating characteristic curve reveals the specificity and sensitivity of GdCho-Len-PET and GdCho-PET in diagnosing patients with lung cancer. GdCho-Len-PET, $\mathrm{Gd}_{2} \mathrm{O}_{3}$-doped carbon-11-choline-lenvatinib nanoparticles contrast combined with positron emission tomography-computed tomography; GdCho-PET, $\mathrm{Gd}_{2} \mathrm{O}_{3}$-doped carbon-11-choline nanoparticles contrast combined with positron emission tomography-computed tomography.

that there were 136 patients with lung cancer among 152 lung cancer patients diagnosed by GdCho-Len-PET, and there were two lung cancer cases in 20 non-lung cancer cases diagnosed by GdCho-Len-PET (data not shown).
Histopathological analyses revealed that there were 114 'true' lung cancer cases in 130 lung cancer cases diagnosed by GdCho-PET. This revealed that there were 102 patients with confirmed lung cancer, as diagnosed by GdCho-PET. There were 21 patients with false positive cases as diagnosed by GdCho-Len-PET, and 28 patients were false positive cases diagnosed by GdCho-PET. In addition, there were five false negative cases diagnosed by GdCho-Len-PET, while there were 34 false negative cases diagnosed by GdCho-PET (Table III). These outcomes indicate that GdCho-Len-PET exhibits higher accuracy compared with GdCho-PET in diagnosing patients with lung cancer.

Plasma concentrations of GdCho-Len in patients with lung cancer. The pharmacodynamics of GdCho-Len was analyzed in patients with lung cancer. The results revealed that GdCho-Len was metabolized from the blood $16 \mathrm{~h}$ following injection (Fig. 5). The clinical data suggested that GdCho-Len is a safe contrast agent when diagnosing patients with lung cancer.

Outcomes for patients diagnosed by GdCho-Len-PET. GdCho-Len-PET contributed to the anticancer treatments in 56 out of $62(90.3 \%)$ patients with lung cancer who were candidates for radiation therapy, 52 out of 57 (91.2\%) patients undergoing adjuvant radiotherapy, and 13 out of 17 (76.5\%) patients undergoing comprehensive therapy (Table IV). Patients diagnosed by GdCho-Len-PET had a significantly 
Table III. Diagnostic efficacy of GdCho-Len-PET for patients suspected of having lung cancer.

\begin{tabular}{lccc}
\hline Result & GdCho-PET, n (\%) & GdCho-Len-PET, n (\%) & P-value \\
\hline False positive & $28(16.3)$ & $21(12.2)$ & 0.030 \\
True positive & $102(59.3)$ & $131(76.2)$ & 0.017 \\
False negative & $34(19.8)$ & $5(2.9)$ & 0.001 \\
True negative & $8(4.7)$ & $15(8.7)$ & 0.0026
\end{tabular}

GdCho-Len-PET, $\mathrm{Gd}_{2} \mathrm{O}_{3}$-doped carbon-11-choline-lenvatinib nanoparticles contrast combined with positron emission tomography-computed tomography; GdCho-PET, $\mathrm{Gd}_{2} \mathrm{O}_{3}$-doped carbon-11-choline nanoparticles contrast combined with positron emission tomography-computed tomography.
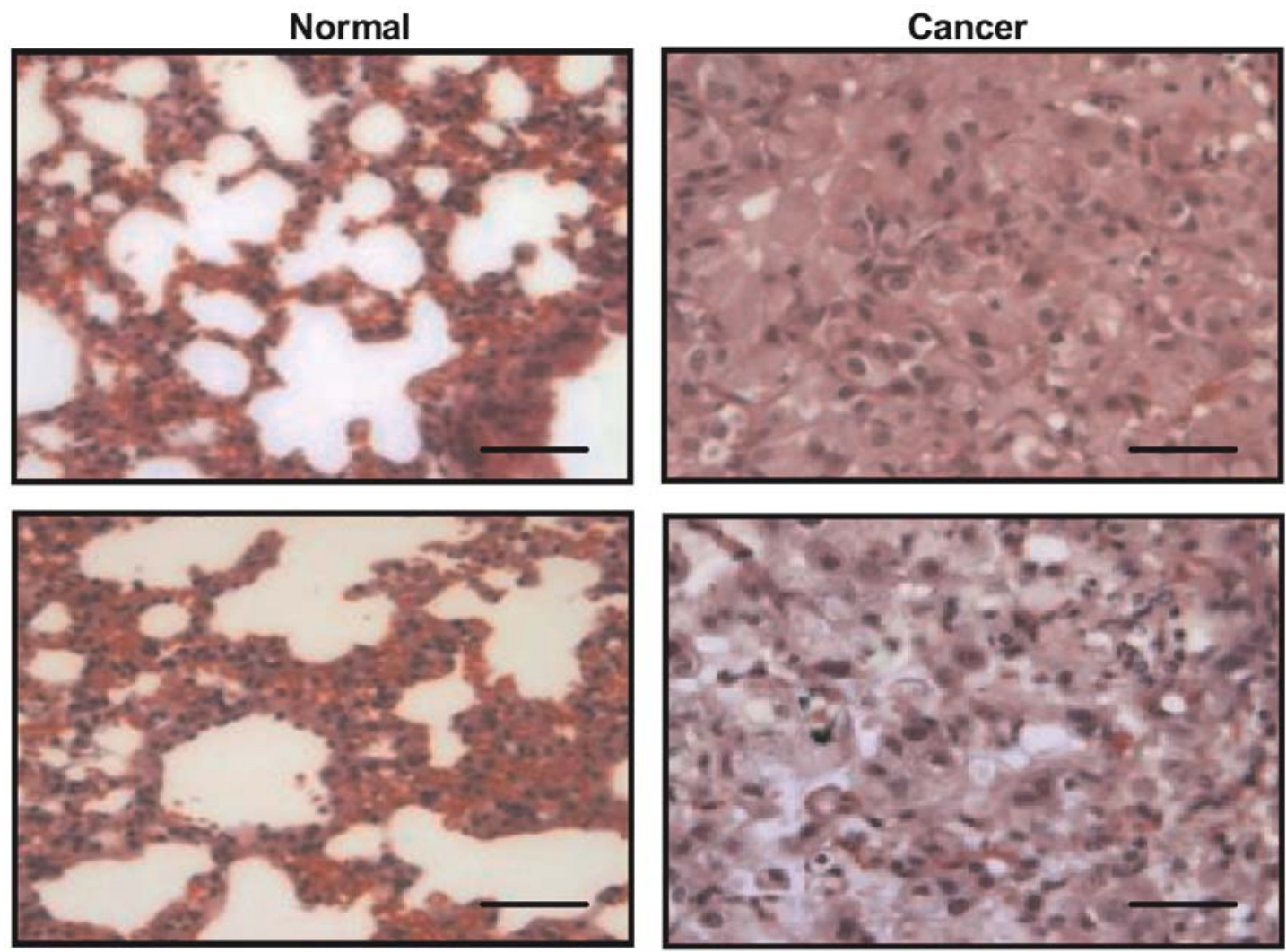

Figure 4. Histopathology confirms the diagnostic accuracy of GdCho-Len-PET for patients with lung cancer. Hematoxylin and eosin staining demonstrates the normal lung tissues and lung cancer tissues. Magnification, $\mathrm{x} 40$. Scale bar, $50 \mu \mathrm{m}$. GdCho-Len-PET, Gd $\mathrm{O}_{3}$-doped carbon-11-choline-lenvatinib nanoparticles contrast combined with positron emission tomography-computed tomography.

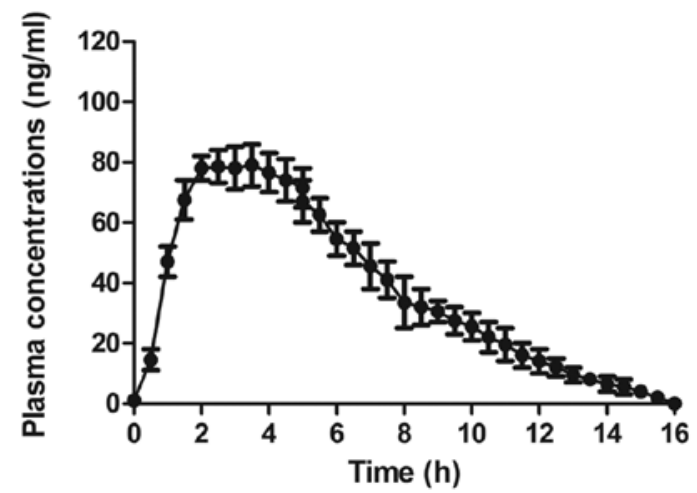

Figure 5. Plasma concentration of GdCho-Len in patients with lung cancer. An ELISA assay revealed that GdCho-Len is metabolized from plasma within $16 \mathrm{~h}$. GdCho-Len, $\mathrm{Gd}_{2} \mathrm{O}_{3}$-doped carbon-11-choline-lenvatinib nanoparticles contrast. improved mean overall survival during the 420-day follow up (Fig. 6A). It was observed that GdCho-Len-PET-diagnosed patients exhibited a significantly improved mean progression-free survival compared with the mean 5-year survival (Fig. 6B). The results demonstrated that 82 patients were alive and tumor-free, 14 patients were still alive with tumors, and 6 patients succumbed to the disease during the 420-day follow-up. These data suggested that patients with lung cancer diagnosed by GdCho-Len-PET had longer median overall survival times compared with the mean 5-year survival.

\section{Discussion}

Lung cancer diagnosis is crucial for reducing morbidity and increasing the quality of life of patients $(24,31,32)$. An early 

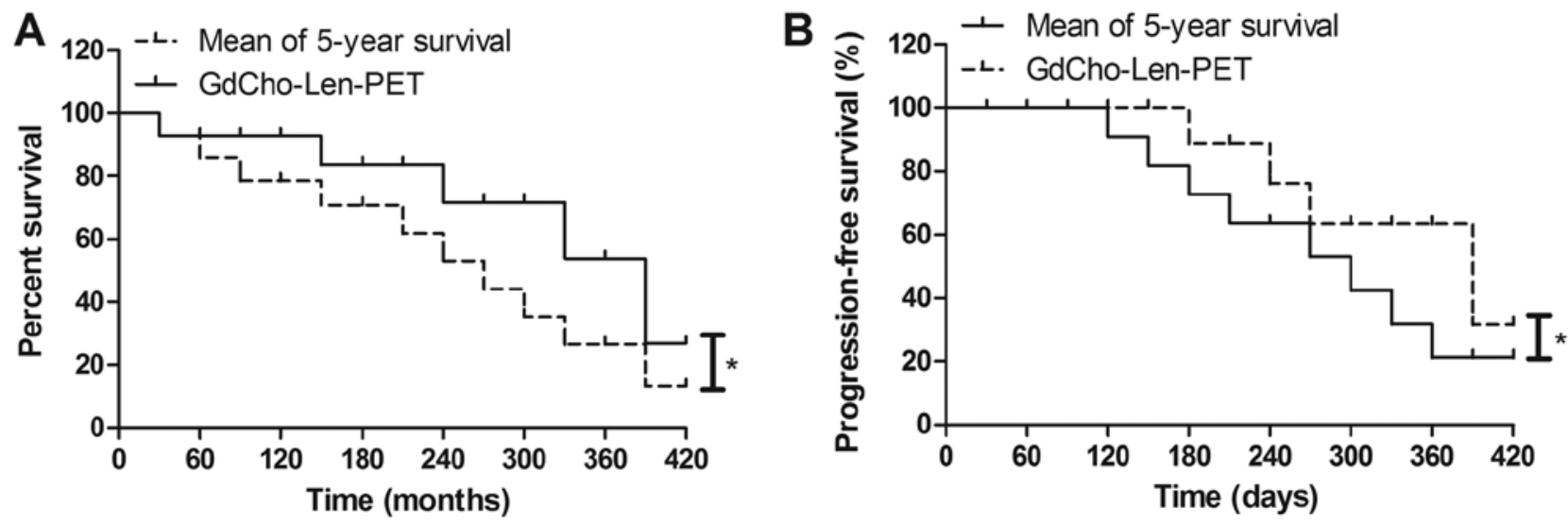

Figure 6. Survival of patients diagnosed by GdCho-Len-PET. (A) Kaplan-Meier curves compare the mean survival rate between patients diagnosed by GdCho-Len-PET and the mean 5-year survival time (log-rank test, $\mathrm{P}=0.035$ ). (B) Kaplan-Meier curves for the mean progression-free survival revealed a significant difference between patients diagnosed by GdCho-Len-PET and the mean 5-year survival (log-rank test, $\mathrm{P}=0.026)$. ${ }^{*} \mathrm{P}<0.05$. GdCho-Len-PET, $\mathrm{Gd}_{2} \mathrm{O}_{3}$-doped carbon-11-choline-lenvatinib nanoparticles contrast combined with positron emission tomography-computed tomography.

Table IV. Treatment of patients with lung cancer diagnosed by GdCho-Len-PET.

\begin{tabular}{lc}
\hline Treatment & $\mathrm{n}(\%)$ \\
\hline Radiation therapy & $56(42.7)$ \\
Adjuvant radiotherapy & $52(39.7)$ \\
Comprehensive therapy & $23(17.6)$ \\
\hline
\end{tabular}

diagnosis of lung cancer may improve the administration of timely anticancer treatments, including surgery, chemoradiotherapy and immunotherapy, for patients with lung cancer, which can further improve the overall survival and progression-free survival (33-35). Clinically, PET/CT has been widely used for diagnosing human lung cancer and evaluating metastatic lesions (36). Previous studies have indicated that contrast agent is useful in PET/CT scanning of human lung cancer (37-39). In the present study, the nanoparticle contrast agent GdCho-Len was administered and the diagnostic efficacy of GdCho-Len-PET was investigated in a total of 172 patients with lung cancer. GdCho-Len-PET provided a $13.8 \%$ false positive result in 152 cases. All cases excluded by GdCho-Len-PET were patients without lung cancer. Taken together, the data obtained in the present study indicates that GdCho-Len is a stable and safe nanoparticle contrast agent for diagnosing patients with lung cancer.

Contrast agent may increase the sensitivity and accuracy of CT imaging for the diagnosis of early stage NSCLC (23). A novel nano-sized chistosan $/ \mathrm{Fe}_{3} \mathrm{O}_{4}$-enclosed bispecific antibody had been identified as an efficient contrast agent in lung cancer diagnosis (40). However, a previous study reported that a nonionic intravenous contrast agent did not cause clinically significant improvement to 18F-FDG PET/CT in patients with lung cancer (41). Therefore, efficient nanoparticles contrast agent serves an important role in diagnosing patients with lung cancer. In the present study, successful encapsulation of Len into the GdCho was achieved and GdCho-Len was produced, which was a stable nanoparticle contrast agent. GdCho-Len exhibited an increased accuracy and sensitivity when compared with GdCho-PET in diagnosing patients suspected of having lung cancer. Indeed, the GdCho-Len nanoparticles provided an improved resolution ratio for tumors than GdCho due to the targeting of Len for tumor cells (42).

Apart from the intracellular environment of lung tumor cells influencing the relaxivity of GdCho-Len, detection of lung tumor cells was difficult to see on the imaging volume within which these cells were embedded (43-45). The present study indicated that the GdCho-Len allowed Len to discriminate between lung cancer cells, which enhanced the diagnostic sensitivity of PET/CT. Ideally, following detection of a suspicious lesion on PET/CT, a plasma metabolic profile of contrast agent could be used to evaluate the clinical safety of drugs (46-48). The current study indicated that GdCho-Len could be metabolized from blood $36 \mathrm{~h}$ post-injection. In addition, GdCho-Len-PET contributed to the anticancer treatments for patients with lung cancer, which further improved the median overall survival and median progression-free survival compared with the mean of 5-year survival. However, further studies that investigate the effect GdCho-Len-PET on radiotherapy should be performed with more patients with lung cancer in the future.

In conclusion, the present study is a clinical report describing the characteristics of GdCho-Len and the diagnostic efficacy of GdCho-Len-PET in patients with suspected lung cancer. The results indicated that GdCho-Len-PET contributed to the anticancer treatments and improved the survival of patients with lung cancer. The results of the current study may aid the diagnosis of lung cancer and the development of effective treatment strategies.

\section{Acknowledgements}

Not applicable.

\section{Funding}

No funding was received. 


\section{Availability of data and materials}

The datasets used and/or analyzed during the study are available from the corresponding author on reasonable request.

\section{Authors' contributions}

TZ, DH and YL performed experiments. JZ, HYW and HGW analyzed experimental data. ET and JY designed the current study and wrote the manuscript.

\section{Ethics approval and consent to participate}

The Ethical Committee of the Dongzhimen Hospital of Beijing University of Traditional Chinese Medicine (Beijing, China) approved the present study. Written informed consent was obtained from all participants.

\section{Patient consent for publication}

Not applicable.

\section{Competing interests}

The authors declare that they have no competing interests.

\section{References}

1. Ridge CA and Boiselle PM: Optimizing the lung cancer screening interval: The world is waiting. J Thorac Dis 8: E1369-E1370, 2016.

2. Xing P, Wang S, Hao X, Zhang T and Li J: Clinical data from the real world: Efficacy of Crizotinib in Chinese patients with advanced ALK-rearranged non-small cell lung cancer and brain metastases. Oncotarget 7: 84666-84674, 2016.

3. Gao L, Xie S, Liu H, Liu P, Xiong Y, Da J, Que C, Dai H and Wang C: Lung cancer in patients with combined pulmonary fibrosis and emphysema revisited with the 2015 World Health Organization classification of lung tumors. Clin Respir J 12: 652-658, 2018.

4. Zhukovsky M, Varaksin A and Pakholkina O: Statistical analysis of observational study of the influence of radon and other risk factors on lung cancer incidence. Radiat Prot Dosimetry 160: 108-111, 2014

5. Torre LA, Bray F, Siegel RL, Ferlay J, Lortet-Tieulent J and Jemal A: Global cancer statistics, 2012. CA Cancer J Clin 65: 87-108, 2015.

6. Bray F, Ferlay J, Soerjomataram I, Siegel RL, Torre LA and Jemal A: Global Cancer Statistics 2018: GLOBOCAN estimates of incidence and mortality worldwide for 36 cancers in 185 countries. CA Cancer J Clin 68: 394-424, 2018.

7. Liu B, Yuan M, Sun Y, Cheng Z, Zhang Z, Hou S, Wang X and Liu J: Incidence and risk of hepatic toxicities associated with anaplastic lymphoma kinase inhibitors in the treatment of non-small-cell lung cancer: A systematic review and meta-analysis. Oncotarget 9: 9480-9488, 2017.

8. Leitinger M, Varosanec MV, Pikija S, Wass RE, Bandke D, Weis S, Studnicka M, Grinzinger S, McCoy MR, Hauer L and Sellner J: Fatal necrotizing encephalopathy after treatment with nivolumab for squamous non-small cell lung cancer: Case report and review of the literature. Front Immunol 9: 108, 2018.

9. Yang JC, Mok T, Han B, Orlando M, Puri T and Park K: A review of regimens combining pemetrexed with an epidermal growth factor receptor tyrosine kinase inhibitor in the treatment of advanced nonsquamous non-small-cell lung cancer. Clin Lung Cancer 19: 27-34, 2018

10. Takamori S, Toyokawa G, Takada K, Shoji F, Okamoto T and Maehara Y: Combination therapy of radiotherapy and Anti-PD-1/PD-L1 treatment in non-small-cell lung cancer: A Mini-review. Clin Lung Cancer 19: 12-16, 2018.
11. Bamji-Stocke S, van Berkel V, Miller DM and Frieboes HB: A review of metabolism-associated biomarkers in lung cancer diagnosis and treatment. Metabolomics 14: 81, 2018.

12. Vergnenègre $\mathrm{A}$ and Chouaïd $\mathrm{C}$ : Review of economic analyses of treatment for non-small-cell lung cancer (NSCLC). Expert Rev Pharmacoecon Outcomes Res 18: 519-528, 2018.

13. Yang X, Li M, Yang X, Zhao M, Huang Y, Dai X, Jiang T, Feng M, Zhan C and Wang Q: Uniport versus multiport video-assisted thoracoscopic surgery in the perioperative treatment of patients with T1-3N0M0 non-small cell lung cancer: A systematic review and meta-analysis. J Thorac Dis 10: 2186-2195, 2018.

14. Suzuki H, Hyodo I and Hasegawa Y: Prediction of decannulation, oral intake recovery, overall survival and lung metastasis following oral malignant tumor resection and reconstruction. Oncol Lett 15: 2686-2694, 2018.

15. Deng Y, Yang Y, Yao B, Ma L, Wu Q, Yang Z, Zhang L and Liu B: Paracrine signaling by VEGF-C promotes non-small cell lung cancer cell metastasis via recruitment of tumor-associated macrophages. Exp Cell Res 364: 208-216, 2018.

16. Song W, Kuang J, Li CX, Zhang M, Zheng D, Zeng X, Liu C and Zhang XZ: Enhanced immunotherapy based on photodynamic therapy for both primary and lung metastasis tumor eradication. ACS Nano 12: 1978-1989, 2018.

17. Du Q, Yu R, Wang H, Yan D, Yuan Q, Ma Y, Slamon D, Hou D, Wang H and Wang Q: Significance of tumor-associated autoantibodies in the early diagnosis of lung cancer. Clin Respir J 12: 2020-2028, 2018.

18. Cormio A, Cormio G, Musicco C, Sardanelli AM, Gasparre G and Gadaleta MN: Mitochondrial changes in endometrial carcinoma: Possible role in tumor diagnosis and prognosis (review). Oncol Rep 33: 1011-1018, 2015.

19. Cherkashin M, Aniskhin M, Berezina N and Puchkov D: CT and PET/CT fusion for lung cancer biopsy planning. BMJ Case Rep 2017: pii: bcr-2017-221972, 2017.

20. Lim CG, Shin KM, Lim JS, Lim JK, Kim HJ, Kim WH, Cho SH, Cha SI, Lee EB, Seock Y and Jeong SY: Predictors of conversion to thoracotomy during video-assisted thoracoscopic surgery lobectomy in lung cancer: Additional predictive value of FDG-PET/CT in a tuberculosis endemic region. J Thorac Dis 9: 2427-2436, 2017.

21. Gensheimer MF, Hong JC, Chang-Halpenny C, Zhu H, Eclov NCW, To J, Murphy JD, Wakelee HA, Neal JW, Le QT, et al: Mid-radiotherapy PET/CT for prognostication and detection of early progression in patients with stage III non-small cell lung cancer. Radiother Oncol 125: 338-343, 2017.

22. Wu Q, Luo W, Zhao Y, Xu F and Zhou Q: The utility of 18F-FDG PET/CT for the diagnosis of adrenal metastasis in lung cancer: A PRISMA-compliant meta-analysis. Nucl Med Commun 38: 1117-1124, 2017.

23. Yuan N, Zhang X, Cao Y, Jiang X, Zhao S, Feng Y, Fan Y, $\mathrm{Lu} \mathrm{Z}$ and Gao H: Contrast-enhanced computerized tomography combined with a targeted nanoparticle contrast agent for screening for early-phase non-small cell lung cancer. Exp Ther Med 14: 5063-5068, 2017.

24. Li N, Han L and Jing H: Contrast-enhanced ultrasound with a novel nanoparticle contrast agent for clinical diagnosis in patients with non-small cell lung cancer. Exp Ther Med 14: 3768-3773, 2017.

25. Nicholls FJ, Rotz MW, Ghuman H, MacRenaris KW, Meade TJ and Modo M: DNA-gadolinium-gold nanoparticles for in vivo T1 MR imaging of transplanted human neural stem cells. Biomaterials 77: 291-306, 2016.

26. Xiao L, Tian X, Harihar S, Li Q, Li L, Welch DR and Zhou A: $\mathrm{Gd}_{2} \mathrm{O}_{3}$-doped silica@Au nanoparticles for in vitro imaging cancer biomarkers using surface-enhanced Raman scattering. Spectrochim Acta A Mol Biomol Spectrosc 181: 218-225, 2017.

27. Deng H, Chen F, Yang C, Chen M, Li L and Chen D: Effect of $\mathrm{Eu}$ doping concentration on fluorescence and magnetic resonance imaging properties of $\mathrm{Gd}_{2} \mathrm{O}_{3}: \mathrm{Eu}^{3+}$ nanoparticles used as dual-modal contrast agent. Nanotechnology 29: 415601, 2018.

28. Zhang H, Wang T, Zheng Y, Yan C, Gu W and Ye L: Comparative toxicity and contrast enhancing assessments of $\mathrm{Gd}_{2} \mathrm{O}_{3} @ B S A$ and $\mathrm{MnO}_{2} @ \mathrm{BSA}$ nanoparticles for MR imaging of brain glioma. Biochem Biophys Res Commun 499: 488-492, 2018.

29. Wirth LJ, Tahara M, Robinson B, Francis S, Brose MS, Habra MA, Newbold K, Kiyota N, Dutcus CE, Mathias E, et al: Treatment-emergent hypertension and efficacy in the phase 3 Study of (E7080) lenvatinib in differentiated cancer of the thyroid (SELECT). Cancer 124: 2365-2372, 2018. 
30. Shao Y, Tian X, Hu W, Zhang Y, Liu H, He H, Shen Y, Xie F and $\mathrm{Li} \mathrm{L}$ : The properties of $\mathrm{Gd} 2 \mathrm{O} 3$-assembled silica nanocomposite targeted nanoprobes and their application in MRI. Biomaterials 33: 6438-6446, 2012.

31. Wojcik E and Kulpa JK: Pro-gastrin-releasing peptide (ProGRP) as a biomarker in small-cell lung cancer diagnosis, monitoring and evaluation of treatment response. Lung Cancer (Auckl) 8: 231-240, 2017.

32. Reck M and Rabe KF: Precision diagnosis and treatment for advanced non-small-cell lung cancer. NEngl J Med 377: 849-861, 2017.

33. Zhou GH, Yang WH and Sun B: Clinical impact of serum miR-661 in diagnosis and prognosis of non-small cell lung cancer. Eur Rev Med Pharmacol Sci 21: 5696-5701, 2017.

34. Labbé C, Anderson M, Simard S, Tremblay L, Laberge F, Vaillancourt R and Lacasse Y: Wait times for diagnosis and treatment of lung cancer: A single-centre experience. Curr Oncol 24: 367-373, 2017.

35. Tzouvelekis A, Spagnolo P, Bonella F, Vancheri C, Tzilas V, Crestani B, Kreuter M and Bouros D: Patients with IPF and lung cancer: Diagnosis and management. Lancet Respir Med 6: 86-88, 2018.

36. Li Y, Jin G and Su D: Comparison of Gadolinium-enhanced MRI and 18FDG PET/PET-CT for the diagnosis of brain metastases in lung cancer patients: A meta-analysis of 5 prospective studies. Oncotarget 8: 35743-35749, 2017.

37. Wang $\mathrm{H}$, Machtaler $\mathrm{S}$, Bettinger T, Lutz AM, Luong R, Bussat $\mathrm{P}$, Gambhir SS, Tranquart F, Tian L and Willmann JK: Molecular imaging of inflammation in inflammatory bowel disease with a clinically translatable dual-selectin-targeted US contrast agent: Comparison with FDG PET/CT in a mouse model. Radiology 267: 818-829, 2013.

38. Aschoff P, Plathow C, Beyer T, Lichy MP, Erb G, Öksüz MÖ, Claussen CD and Pfannenberg C: Multiphase contrast-enhanced CT with highly concentrated contrast agent can be used for PET attenuation correction in integrated PET/CT imaging. Eur J Nucl Med Mol Imaging 39: 316-325, 2012.

39. Hyafil F, Cornily JC, Rudd JH, Machac J, Feldman LJ and Fayad ZA: Quantification of inflammation within rabbit atherosclerotic plaques using the macrophage-specific CT contrast agent N1177: A comparison with 18F-FDG PET/CT and histology. J Nucl Med 50: 959-965, 2009.

40. Gao J, Li L, Liu X, Guo R and Zhao B: Contrast-enhanced magnetic resonance imaging with a novel nano-size contrast agent for the clinical diagnosis of patients with lung cancer. Exp Ther Med 15: 5415-5421, 2018.
41. An YS, Sheen SS, Oh YJ, Hwang SC and Yoon JK: Nonionic intravenous contrast agent does not cause clinically significant artifacts to $18 \mathrm{~F}-\mathrm{FDG}$ PET/CT in patients with lung cancer. Ann Nucl Med 21: 585-592, 2007.

42. Nishio M, Horai T, Horiike A, Nokihara H, Yamamoto N, Takahashi T, Murakami H, Yamamoto N, Koizumi F, Nishio K, et al: Phase 1 study of lenvatinib combined with carboplatin and paclitaxel in patients with non-small-cell lung cancer. Br J Cancer 109: 538-544, 2013.

43. Wu Y, Zhang H, Xiang J, Mao Z, Shen G, Yang F, Liu Y, Wang W, Du N, Zhang J and Tang Y: Ultrasensitive and high specific detection of non-small-cell lung cancer cells in human serum and clinical pleural effusion by aptamer-based fluorescence spectroscopy. Talanta 179: 501-506, 2018.

44. Cai NL, Lau ATY, Yu FY, Wu DD, Dai LJ, Mo HY, Lin CM and $\mathrm{Xu}$ YM: Purification and characterization of a highly specific polyclonal antibody against human extracellular signal-regulated kinase 8 and its detection in lung cancer. PLoS One 12: e0184755, 2017.

45. Sun Y, Liu S, Qiao Z, Shang Z, Xia Z, Niu X, Qian L, Zhang Y, Fan L, Cao CX and Xiao H: Systematic comparison of exosomal proteomes from human saliva and serum for the detection of lung cancer. Anal Chim Acta 982: 84-95, 2017.

46. Ohliger MA, von Morze C, Marco-Rius I, Gordon J, Larson PEZ, Bok R, Chen HY, Kurhanewicz J and Vigneron D: Combining hyperpolarized ${ }^{13} \mathrm{C}$ MRI with a liver-specific gadolinium contrast agent for selective assessment of hepatocyte metabolism. Magn Reson Med 77: 2356-2363, 2017.

47. Uran S, Landmark K, Normann PT, Hals PA, Toft KG and Skotland T: A respiration-metabolism chamber system and a GC-MS method developed for studying exhalation of perfluorobutane in rats after intravenous injection of the ultrasound contrast agent Sonazoid. J Pharm Biomed Anal 39: 746-751, 2005.

48. Skotland T, Sontum PC and Oulie I: In vitro stability analyses as a model for metabolism of ferromagnetic particles (Clariscan), a contrast agent for magnetic resonance imaging. J Pharm Biomed Anal 28: 323-329, 2002.

This work is licensed under a Creative Commons Attribution-NonCommercial-NoDerivatives 4.0 International (CC BY-NC-ND 4.0) License. 